\title{
Mortality, complications and long-term functional outcome in elderly patients with fragility fractures of the acetabulum
}

Johannes Wollmerstädt, Philipp Pieroh, Isabell Schneider, Suzanne Zeidler, Andreas Höch, Christoph Josten and Georg Osterhoff*

\begin{abstract}
Background: Early operative treatment of acetabulum fractures in geriatric patients has been suggested to reduce pain and allow for earlier mobilization. The aim of this study was to determine mortality, complications and functional outcome after operative and non-operative treatment.

Methods: Patients aged $\geq 60$ years with operative treatment of low-energy fragility fracture of the acetabulum from 2009 to 2016 and a follow-up of at least 24 months were identified. The patients were contacted by phone and a modified Merle d'Aubigné score was obtained. If patients or their relatives were not available for follow-up, mortality data was assessed using a national social insurance database.

Results: One hundred seventy-six patients (mean age 78, SD 10 years; 73 female) were available for analysis of mortality data. At final follow-up (68 months, SD 26, range, 24 to 129), 99/176 patients (56.3\%) had deceased. Oneyear-mortality was $25.0 \%$ and 2-year mortality $35.8 \%$. Type of treatment (non-operative vs. operative) did not affect mortality at 1 and 2 years ( $p=.65$ and $p=.10)$. Hospital-acquired infections were observed in 31/176 cases (17.6\%), thromboembolic events and delirium in 6 patients (3.4\%). In-hospital mortality was $5.7 \%$. Patients who underwent operative treatment were more likely to have an in-hospital infection $(p=.02)$ but less likely to sustain thromboembolic events ( $p=.03$ ).

The mean hospital stay was 14 days (SD 10 days, range, 1 to 66). Patients with operative treatment were longer hospitalized than patients with non-operative treatment $(p<.001)$.

The rate of secondary conversions to THA was $12.4 \%$, this was not affected by initial treatment.

The mean modified Merle d'Aubigné Score of those patients available for a final follow-up ( $n=47$; follow-up 56 months, SD 28, range, 24 to 115) was 14/18 points, SD 3 (range 7 to 18). Functional results at final follow-up between operatively and non-operatively treated patients were without difference.

Conclusions: All-cause mortality and in-hospital complications are high among geriatric patients with low-energy fractures of the acetabulum even when treated operatively. Secondary conversion rates to THA are similar to those seen in younger patients. Mid-term functional outcome in those surviving is fair.
\end{abstract}

Keywords: Geriatric, Acetabulum, Mortality, Functional outcome, Non-operative, Complications, Fracture

\footnotetext{
* Correspondence: georg.osterhoff@medizin.uni-leipzig.de

Department of Orthopaedics, Trauma and Plastic Surgery, University Hospital
} Leipzig, 04103 Leipzig, Germany

(c) The Author(s). 2020 Open Access This article is distributed under the terms of the Creative Commons Attribution 4.0 International License (http://creativecommons.org/licenses/by/4.0/) which permits unrestricted use, distribution, and reproduction in any medium, provided you give appropriate credit to the original author(s) and the source, provide a link to the Creative Commons license, and indicate if changes were made. The Creative Commons Public Domain Dedication waiver (http://creativecommons.org/publicdomain/zero/1.0/) applies to the data made available in this article, unless otherwise stated. 


\section{Background}

Over the past decades andwith an ageing population, fragility fractures of the acetabulum have become more common and thus a greater concern [1].

A nationwide study covering the population of Finland, reported an increase by $30 \%$ in the incidence of acetabular fractures in individuals aged older than 65 within the past 20 years, with the incidence now being 23/100,000 per year [2]. Ferguson et al. even found a more than twofold increase amongst North American patients beyond the age of 60 within a similar time period [3].

Whereas the main mechanism of injury for younger patients is high-energy-trauma, the majority of acetabular fractures in the elderly are a result of a fall from standing height [2-5].

Like in geriatric fractures of the hip, the very similar impact of acetabular fractures on walking ability has the potential of high morbidity and mortality due to immobilization and complications associated with bedrest.

The treatment of displaced acetabular fractures in younger patients usually consists of open reduction and internal fixation, but since most of the elderly patients present with various comorbidities and decreased bone quality, an individualized treatment approach is necessary. Furthermore, patients of greater age are in need of early mobilization with regard to these comorbidities, and usually cannot follow partial weight-bearing protocols [6]. Treatment may vary from non-operative treatment, open reduction and internal fixation, minimally invasive percutaneous screw fixation to even primary total arthroplasty of the hip [7, 8].

It has been suggested, that operative treatment of these fractures may reduce pain, allow for earlier mobilization and shorter hospitalization time, thus leading to less associated complications $[8,9]$. In contrast, as suggested by a smaller cohort study, operative treatment may also lead to more conversions to total hip arthroplasty [10].

It remains unclear, whether these potential short-term benefits also translate into better functional outcome and a lower mortality rate and would accordingly justify the perioperative risks of a surgical intervention.

The aim of this study was therefore to assess mid- to long-term mortality and functional outcome after geriatric acetabular fractures and to compare the outcome after surgical and conservative treatment in elderly patients.

\section{Methods}

\section{Patients}

The study protocol of this study was approved by the institutional ethics committee. Consecutive patients aged $\geq 60$ years who had operative or conservative treatment of a low-energy fracture of the acetabulum between January 2008 and December 2016 with a follow-up of at least 24 months were identified from a prospective database of patients with acetabular fractures. The database is kept at our institution as part of the German Pelvis Registry and includes epidemiologic data and data on fracture patterns, therapy and in-hospital complications. For all patients identified from this database, a retrospective chart review was performed and eligibility was assessed. Exclusion criteria included the presence of an additional fracture of the lower extremity or pelvis and pathological fractures. Also, patients who had expressed objection to the use of their data for research purposes were excluded.

By chart review, additional information on comorbidities (expressed as ASA - American Society of Anesthesiologists Scale), complications and revision surgery was obtained. Mortality data was assessed using the national statutory pension insurances database. Patients, eligible according to the inclusion and exclusion criteria, were contacted by phone and after acquiring informed consent, functional outcome was assessed by the modified Merle d'Aubigné Score [11]. If the patients could not be contacted by a first phone call, two more attempts were made by phone and mail. Informed consents were acquired by phone and archived as mp3 audio files.

If patients were not able to understand the Merle d'Aubigné questionnaire due to language or cognitive impairment, they were excluded from the functional outcome assessment. In case personal contact could not be established, family doctors were contacted to assess mortality data and data on the conversion to total hip arthroplasty (THA).

\section{Outcome}

Primary outcome was the all-cause mortality rate at 1 and 2 years. Survival was assessed through the national social insurance database (Deutsche Rentenversicherung) that provides mortality data for every German resident who received statutory pension.

Secondary outcomes were in-hospital complications, duration of the hospitalization, and secondary conversion to THA. In those patients who had survived the follow-up, functional outcome was assessed by a modified Merle d'Aubigné Score through telephone interviews.

\section{Statistical analysis}

Statistical analysis was performed with SPSS for windows 25.0 (SPSS, Chicago, Illinois, USA). Data are presented as frequencies (n) and means with range and standard deviation (SD). To assess differences in means between different treatment groups, an independent- 
samples t-test was used for the normally distributed continuous data and a Chi-Square or Fisher's exact test for categorical data. Missing data were reported as such for each outcome parameter. The level of statistical significance was set at $p<0.05$.

\section{Results}

One hundred seventy-six patients $(n=176$; mean age 78 , SD 10 years; 73 female) were available for final analysis of mortality data (Fig. 1). Of those who had survived at the time of data acquisition $(n=77), 47$ patients were available for functional outcome assessment. Mean ASA-Score (American Society of Anesthesiologists Scale) was $2.8 \pm 0.5$. Patients who were treated nonoperatively were more likely to have an ASA score $>2$ (56/67, 83.6\%) when compared to patients who were treated operatively $(75 / 109,68.8 \%, p=.03)$.

In 15 patients, the acetabular fracture occurred in the presence of a total hip implant in terms of a periprosthetic fracture. Of the remaining 161 patients with fractures of the native acetabulum, 67/161 patients (41.6\%) were treated non-operatively, 10 patients $(6.2 \%)$ by percutaneous fixation, 82 patients by open fixation (Fig. 2), and two patients (1.1\%) received THA as primary treatment.

At the time of final follow up (68 months, SD 26, range, 24 to 129$), 99 / 176$ patients $(56.3 \%)$ had deceased. Forty-four patients deceased during the first year after the hospitalization (1-year-mortality: 25\%) and 2-year mortality was $35.8 \%$ (63/176 patients). The type of treatment (non-operative vs. operative) did not affect mortality at 1 and 2 years $(p=.65$ and $p=.10$, Table 1$)$.

Complications during the hospitalization occurred in $41 / 176$ patients (23.2\%). Hospital-acquired infections like surgical-site infections, pneumonia and urinary tract infections were observed in 31/176 cases (17.6\%), thromboembolic events and a delirium were documented each in 6 patients (3.4\%). Ten patients (5.7\%) died during prolonged hospitalizations secondary to pneumonia $(n=4)$, pulmonary embolism $(n=$ $3)$, myocardial infarction $(n=1)$, and delirium $(n=1)$. Patients who underwent operative treatment were more likely to have an in-hospital infection $(p=.02)$ but less likely to sustain thromboembolic events $(p=.03$, Table 1$)$.

The mean duration of the hospitalization was 14 days (SD 10 days, range, 1 to 66). Prior to the acetabulum

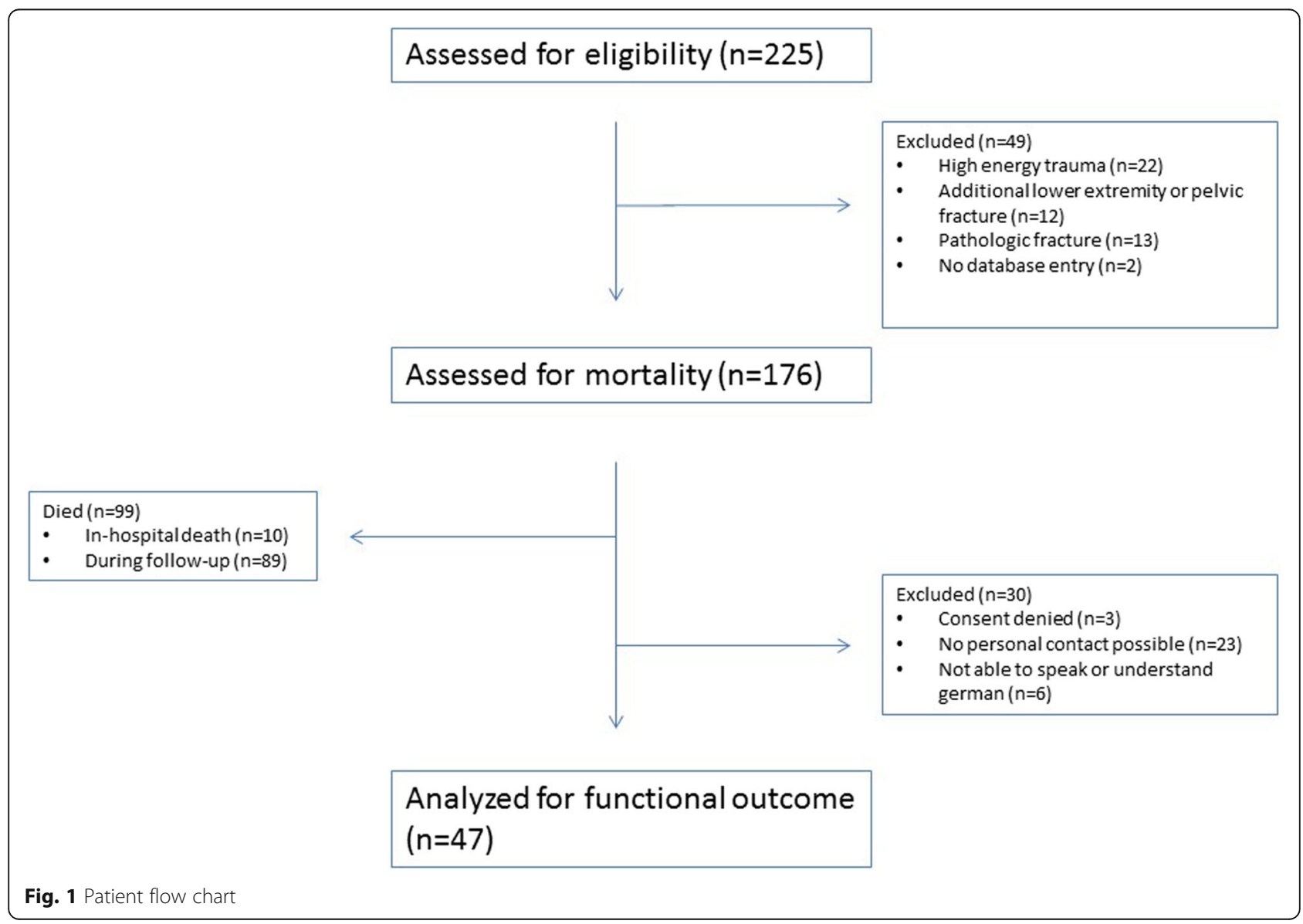




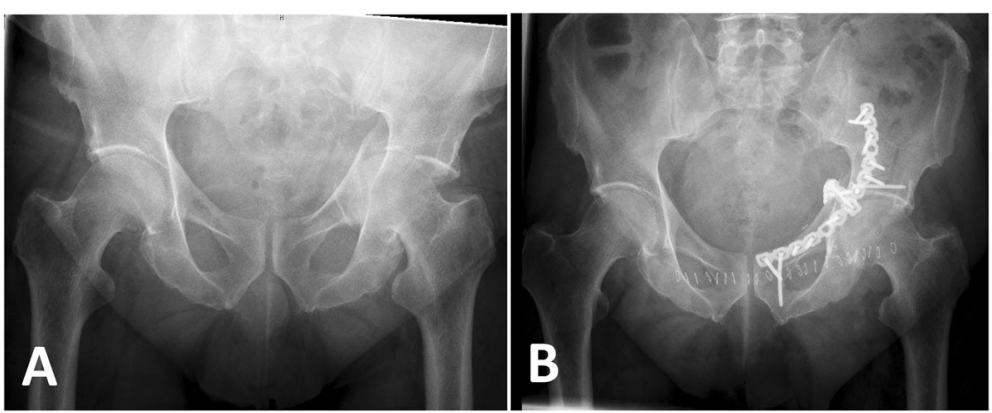

Fig. 2 Case. Seventy-five year-old male patient with a left acetabulum fracture after a fall from standing height. a Pelvis AP radiograph on admission showing the central dislocation of the femoral head into the lesser pelvis. b Pelvis AP radiograph after open reduction and internal plate fixation

fracture, $143 / 176$ patients (81.3) had lived at home (43 with home care). After the fracture, only 93 (65.0\%) of these 143 patients were able to eventually return to their homes (31 with home care). Patients with operative treatment stayed longer in the hospital than patients with non-operative treatment $(p<.001$, Table 1$)$.

Twenty of the 161 patients (12.4\%) with a nonperiprosthetic fracture of the native acetabulum had to undergo secondary conversion to THA for posttraumatic osteoarthritis during follow-up. In the non-operative group, this was necessary in $5 / 67$ patients $(7.5 \%)$ versus in $15 / 92$ patients $(16.3 \%)$ in the operative group $(p=.33)$.

Table 1 Patients' baseline characteristics, complications and survival $(N=176)$

\begin{tabular}{llll}
\hline & Non-operative & Operative & \\
\hline $\mathrm{N}$ & 67 & 109 & \\
Follow-Up [m] & 70, SD 31 & 67, SD 27 & $.505^{\mathrm{a}}$ \\
Age [y] & 80, SD 10 & 77, SD 9 & $.051^{\mathrm{a}}$ \\
Gender [f: m] & $38: 29$ & $35: 74$ & $.002^{\mathrm{b}}$ \\
ASA & & & $.033^{\mathrm{c}}$ \\
1 & 2 & 1 & \\
2 & 9 & 33 & \\
3 & 56 & 72 & \\
4 & 0 & 3 & $<.001^{\mathrm{a}}$ \\
Hospitalization duration [d] & 8, SD 6 & 17, SD 10 & \\
In-hospital complications [n (\%)] & $6(9.0 \%)$ & $25(22.9 \%)$ & $.024^{\mathrm{b}}$ \\
Infection & $5(7.5 \%)$ & $1(0.9 \%)$ & $.030^{\mathrm{b}}$ \\
$\quad$ Thromboembolic event & $1(1.5 \%$ & $5(4.6 \%)$ & $.410^{\mathrm{b}}$ \\
Delirium & $4(6.0 \%)$ & $6(5.5 \%)$ & $1.00^{\mathrm{b}}$ \\
In-hospital death & $18(26.9 \%)$ & $26(23.9 \%)$ & $.654^{\mathrm{b}}$ \\
1-year mortality & $29(43.3 \%)$ & $34(31.2 \%)$ & $.104^{\mathrm{b}}$ \\
2-year mortality & & & \\
\hline
\end{tabular}

${ }^{\mathrm{a}}$ Student's T-test. ${ }^{\mathrm{b}}$ Pearson Chi-square/Fisher's test

'Pearson Chi-square test for ASA $\leq 2$ vs. ASA $>2$. There were no patients with $\mathrm{ASA}>4$
The mean modified Merle d'Aubigné Score of those patients who had survived and were available for a final follow-up by phone ( $n=47$; follow-up 56 months, SD 28, range, 24 to 115 ) was $14 / 18$ points, SD 3 , range 7 to 18 ). Functional results at final follow-up were not different between survivors who had been treated operatively and non-operatively (Table 2).

\section{Discussion}

The aim of this study was to evaluate mortality and functional outcome of patients with geriatric fracture of the acetabulum, more than 2 years after treatment.

It was shown, that in this cohort of geriatric patients with fragility fractures of the acetabulum, all-cause mortality and in-hospital complications were high, regardless whether the patients underwent non-operative or operative treatment. While operatively treated patients were more susceptible to infection, thromboembolic events occurred more often in the non-operatively treated group. The conversion-rate to secondary total hip arthroplasty was $12.4 \%$, with no significant difference between the groups. Patients surviving until final follow-up showed fair functional outcome and no difference between operative versus non-operative treatment occurred. We found no differences between the operative and the non-operative group in regards to all-cause 1year-mortality. This finding is consistent with a previous studies that conclude that there is no difference in 1-

Table 2 Functional outcome of survivors at final follow-up ( $N=$ 47)

\begin{tabular}{llll}
\hline & Non-operative & Operative & $p$ \\
\hline $\mathrm{N}$ & 12 & 35 & \\
Merle d'Aubigné & 14, SD 3 & 15, SD 3 & $.890^{\mathrm{a}}$ \\
$\quad$ Pain & 5, SD 1 & 5, SD 1 & $.748^{\mathrm{a}}$ \\
Mobility & 5, SD 2 & 5, SD 1 & $.628^{\mathrm{a}}$ \\
Walking & 4, SD 2 & 5, SD 2 & $.763^{\mathrm{a}}$ \\
\hline
\end{tabular}

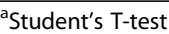


year mortality between operative and non-operative management, once comorbidities are taken into account $[4,10]$.

Existing literature states 1 -year mortality rates of 16 to $35 \%$ for non-operative treatment $[10,12-14]$ and of 4 to $21 \%$ for operative treatment $[10,12,13]$. Reasons for the higher mortality in the present study include the fact that a follow-up of $100 \%$ was achieved for mortality data by the use of the national social insurance database. In addition, only patients with a low-energy trauma were included. Patients older than 60 years of age with acetabular fracture as a result of high energy accidents are possibly in a better state of general health and live a more active lifestyle, than patients whose acetabular fracture is a result of same level falling and thus a representation of comorbidity.

The average duration of hospital stay was long (14 days, SD 10 days), compared to other studies that state a median hospitalisation of 8 to 11 days [10, 14, 15].Operatively treated patients had a longer average hospitalisation time, which is consistent with the findings of one previous publication on this topic [15].

The 47 patients that had survived until final follow up in the present study showed a fair functional outcome and no difference between operatively and non-operatively treated patients. While there seems to be consensus that good functional outcomes can be achieved by operative treatment of acetabular fractures in the elderly [12, 16-18], there is little data available on functional outcome after non-operative treatment. A recent retrospective case series of elderly patients with acetabular fractures that met criteria for surgery found that these patients can have good functional outcome also with non-operative treatment [14].

Secondary conversion rate to THA was $12.4 \%$ in the whole cohort and there was no significant difference between the operative and the non-operative group. Secondary conversion rates to THA have been reported to range from 10 to $37 \%$ [16] for patients in this age group when treated by open reduction and internal fixation and $15 \%$ when treated non-operatively [14]. A recent registry analysis of 678 acetabular fractures among all age groups reported that $19.8 \%$ of the patients required secondary THA [19]. Hence, the conversion rates found in our cohort are comparable to what is found in the literature.

The limitations of this study are inherent with its retrospective design. Therefore, selection bias for operative treatment is possible, since fractures with a less complex morphology were more likely to be assigned to non-operative treatment. Patients in the non-operative group had a higher ASA-Score, illustrating that these patients were in a worse state of general health and less amenable for both, primary and secondary operative interventions. No physical follow-up examinations were performed for functional outcome assessment. However, conducting follow-up interviews by phone enabled us to also assess patients, that were not able to leave their homes or nursing facilities and otherwise could not have been included. Still, we were able to only assess the functional outcome of 47 of 77 surviving patients resulting in a loss to follow-up of $39 \%$. This comes with no surprise with regard to the geriatric population of this study and a follow-up interval of up to 10 years (129 months). Functional outcome was assessed mean 68 months after the trauma and in a small sample of patients who had mainly been treated operatively. This may provide a selection bias towards patients with better function resulting in better survival.

In conclusion, mortality and in-hospital complications remain high among geriatric patients with low-energy fractures of the acetabulum even when treated operatively. Secondary conversion rates to THA are similar to those seen in younger patients. Mid-term functional outcome in those surviving is fair.

Treatment decisions for these fractures in the elderly depend on multiple factors and can only be done individualized. The findings of this study may help surgeons in the decision-making and management of acetabular fractures after low-energy trauma in geriatric patients. The knowledge about the high rate of mortality and complications may guide surgeons towards nonoperative treatment in very old patients with serious comorbidities. It may also be that selected elderly patients with acetabular fractures would benefit from primary THA as this usually allows for earlier mobilisation with full-weight bearing. Future prospective studies with larger cohorts need to further investigate the potential benefits of these different therapeutic strategies.

\section{Conclusions}

All-cause mortality and in-hospital complications remain high among geriatric patients with low-energy fractures of the acetabulum even when treated operatively. Secondary conversion rates to THA are similar to those seen in younger patients. Mid-term functional outcome in those surviving is fair.

\section{Abbreviations}

ASA scale: American Society of Anesthesiologists Scale; SD: Standard deviation; THA: Total hip arthroplasty

\section{Acknowledgements}

Not applicable.

\section{Consent to publish}

$\mathrm{N} / \mathrm{A}$

Authors' contributions

JW contacted all patients by phone and participated in designing the study and drafting the manuscript. PP, IS, SZ and AH participated in designing the study, participated in data acquisition and revised the manuscript. CJ participated in designing the study and revised the manuscript. GO 
participated in designing the study, helped with data acquisition, did the statistical analysis and wrote the first draft of the manuscript. The author(s) read and approved the final manuscript.

\section{Funding}

There is no external funding source.

\section{Availability of data and materials}

Anonymized grouped data available upon request from the corresponding author.

\section{Ethics approval and consent to participate}

The study protocol of this study was approved by the institutional ethics committee (Ethik-Kommission der Medizinischen Fakultät der Universität Leipzig, reference: 169/18-ek). As approved by the ethics committee, consent was given verbally by those patients contacted by phone, and no consent was required for chart review of patients who had deceased or could not be contacted by phone.

\section{Competing interests}

$\mathrm{GO}$ and $\mathrm{AH}$ are Associated Editors of BMC Musculoskeletal Disorders. The other authors declare that they have no conflicts of interest.

\section{Received: 25 October 2019 Accepted: 11 February 2020}

Published online: 17 February 2020

\section{References}

1. Herath SC, Holstein JH, Pizanis A, et al. Azetabulumfrakturen: Komplikationen und Endoprothetik (fractures of the acetabulum: complications and joint replacement). Z Orthop Unfall. 2014;152(4):399-413. https://doi.org/10.1055/ s-0034-1382862.

2. Rinne PP, Laitinen MK, Huttunen $T$, et al. The incidence and trauma mechanisms of acetabular fractures: a nationwide study in Finland between 1997 and 2014. Injury. 2017;48(10):2157-61. https://doi.org/10.1016/j.injury. 2017.08.003.

3. Ferguson TA, Patel R, Bhandari $M$, et al. Fractures of the acetabulum in patients aged 60 years and older: an epidemiological and radiological study. The journal of bone and joint surgery. British volume. 2010;92(2):2507. https://doi.org/10.1302/0301-620X.92B2.22488.

4. Gary $\mathrm{L}$, Paryavi E, Gibbons SD, et al. Effect of surgical treatment on mortality after acetabular fracture in the elderly: a multicenter study of 454 patients. J Orthop Trauma. 2015;29(4):202-8. https://doi.org/10.1097/BOT. 0000000000000223.

5. Laird A, Keating JF. Acetabular fractures: a 16-year prospective epidemiological study. J Bone Joint Surg Br. 2005;87(7):969-73. https://doi. org/10.1302/0301-620X.87B7.16017.

6. Ruckstuhl T, Osterhoff G, Zuffellato M, et al. Correlation of psychomotor findings and the ability to partially weight bear. Sports Med Arthrosc Rehabil Ther Technol. 2012;4:6. https://doi.org/10.1186/1758-2555-4-6.

7. Hessmann MH, Nijs S, Rommens PM. Acetabulumfrakturen im Alter Ergebnisse eines differenzierten Behandlungskonzeptes (Acetabular fractures in the elderly. Results of a sophisticated treatment concept). Unfallchirurg. 2002;105(10):893-900. https://doi.org/10.1007/s00113-002-0437-0.

8. Pagenkopf E, Grose A, Partal G, et al. Acetabular fractures in the elderly: treatment recommendations. HSS J. 2006;2(2):161-71. https://doi.org/10. 1007/s11420-006-9010-7.

9. Vanderschot P. Treatment options of pelvic and acetabular fractures in patients with osteoporotic bone. Injury. 2007;38(4):497-508. https://doi.org/ 10.1016/j.injury.2007.01.021

10. Walley KC, Appleton PT, Rodriguez EK. Comparison of outcomes of operative versus non-operative treatment of acetabular fractures in the elderly and severely comorbid patient. Eur J Orthop Surg Traumatol. 2017; 27(5):689-94. https://doi.org/10.1007/s00590-017-1949-1.

11. Merle d'Aubigne R. Résultats fonctionnels de l'arthroplastie de la hanche (functional results of arthroplasty of the hip). Acta Orthop Belg. 1953;19(3): 81-103.

12. Briffa N, Pearce R, Hill AM, et al. Outcomes of acetabular fracture fixation with ten years' follow-up. The journal of bone and joint surgery. British volume. 2011;93(2):229-36. https://doi.org/10.1302/0301-620X.93B2.24056.

13. O'Toole RV, Hui E, Chandra A, et al. How often does open reduction and internal fixation of geriatric acetabular fractures lead to hip arthroplasty? J
Orthop Trauma. 2014;28(3):148-53. https://doi.org/10.1097/BOT. Ob013e31829c739a.

14. Ryan SP, Manson TT, Sciadini MF, et al. Functional outcomes of elderly patients with nonoperatively treated Acetabular fractures that meet operative criteria. J Orthop Trauma. 2017;31(12):644-9. https://doi.org/10. 1097/BOT.0000000000000990.

15. Bible JE, Wegner A, McClure DJ, et al. One-year mortality after acetabular fractures in elderly patients presenting to a level-1 trauma center. J Orthop Trauma. 2014;28(3):154-9. https://doi.org/10.1097/BOT.0b013e31829e801b.

16. Antell NB, Switzer JA, Schmidt AH. Management of Acetabular Fractures in the elderly. J Am Acad Orthop Surg. 2017;25(8):577-85. https://doi.org/10. 5435/JAAOS-D-15-00510

17. Helfet DL, Borrelli J Jr, Di Pasquale T, et al. Stabilization of acetabular fractures in elderly patients. J Bone Joint Surg Am. 1992;74(5):753-65.

18. Jeffcoat DM, Carroll EA, Huber FG, et al. Operative treatment of acetabular fractures in an older population through a limited ilioinguinal approach. J Orthop Trauma. 2012;26(5):284-9. https://doi.org/10.1097/BOT. Ob013e31821e10a2

19. Rollmann MF, Holstein JH, Pohlemann T, et al. Predictors for secondary hip osteoarthritis after acetabular fractures-a pelvic registry study. Int Orthop. 2018. https://doi.org/10.1007/s00264-018-4169-3.

\section{Publisher's Note}

Springer Nature remains neutral with regard to jurisdictional claims in published maps and institutional affiliations.
Ready to submit your research? Choose BMC and benefit from:

- fast, convenient online submission

- thorough peer review by experienced researchers in your field

- rapid publication on acceptance

- support for research data, including large and complex data types

- gold Open Access which fosters wider collaboration and increased citations

- maximum visibility for your research: over $100 \mathrm{M}$ website views per year

At $\mathrm{BMC}$, research is always in progress.

Learn more biomedcentral.com/submissions 